\title{
Observing the Elusive Double-Peak Structure in R-dependent Tunneling Ionization Rate of Hydrogen Molecular Ion
}

\author{
I.V. Litvinyuk ${ }^{1}$, Han $\mathrm{Xu}^{1}$, Tian-Yu $\mathrm{Xu}^{3}$, Feng $\mathrm{He}^{3}$, D. Kielpinski ${ }^{1,2}$ and R.T. Sang ${ }^{1,2}$ \\ ${ }^{1}$ Centre for Quantum Dynamics and Australian Attosecond Science Facility, Griffith University, Nathan, QLD 4111, Australia \\ ${ }^{2}$ ARC Centre for Coherent X-Ray Science, Griffith University, Nathan, QLD 4111, Australia \\ ${ }^{3}$ Key Laboratory for Laser Plasmas (Ministry of Education), and Department of Physics and Astronomy, SJTU, Shanghai 200240, People's Republic of \\ China \\ i.litvinyuk@griffith.edu.au
}

Abstract: We performed pump-probe experiment on $\mathrm{H}_{2}$ using intense few-cycle laser pulses and Reaction Microscope detection apparatus. We observe the theoretically predicted [1] double-peak structure in R-dependent tunneling ionization rate for the first time experimentally.

OCIS codes: (020.2649) Strong field laser physics; (020.4180) Multiphoton processes

Tunneling ionization of hydrogen molecular ion by intense femtosecond near-infrared laser pulses exhibits strong dependence on internuclear separation R. It has been shown that rate of such ionization increases dramatically when $\mathrm{R}$ reaches certain critical distance $\mathrm{R}_{\mathrm{c}}(\sim$ 7 atomic units). This phenomenon is known as enhanced ionization [2] and it explains kinetic energy release (KER) spectra measured in Coulomb explosion of $\mathrm{H}_{2}$ by femtosecond laser pulses: that kinetic energy reflects the internuclear distance where the second ionization takes place $\left(R_{c}\right)$.

In terms of physical mechanism, enhanced ionization is explained by appearance of charge-resonance states in field-distorted double-Coulomb-well potential of $\mathrm{H}_{2}^{+}$, and therefore it is often referred to as charge-resonanceenhanced ionization or CREI [1]. Similar mechanism is thought to be operational also in larger diatomic, triatomic and even polyatomic molecules.

Theoretical simulations predict that R-dependent ionization rate for $\mathrm{H}_{2}^{+}$should exhibit two well-defined maxima - one at around 7 au and another at around $11 \mathrm{au}$ $[1,3]$. However, only the first maximum manifests itself in experimental KER spectra. The second maximum was never observed experimentally and various explanations were advanced to explain its elusiveness [4].

Here we present for the first time an experimental observation of full double-peak structure of R-dependent ionization rate for $\mathrm{H}_{2}{ }^{+}$, including well-defined second maximum at 12 au. To observe this structure we performed pump-probe measurements on neutral hydrogen molecules using two 6 fs (750 nm central wavelength) laser pulses. Following initial ionization by the pump, we traced KER spectra for the double-proton coincidence channel as a function of pump-probe delay. The experimental timedependent ionization yield displays two clear maxima at 14 and 22 fs (Figure 1). By modeling dynamics of nuclear wavepackets in $\mathrm{H}_{2}^{+}$we determine that those maxima correspond to internuclear separations of 7 and 12 au in excellent agreement with theoretical predictions.
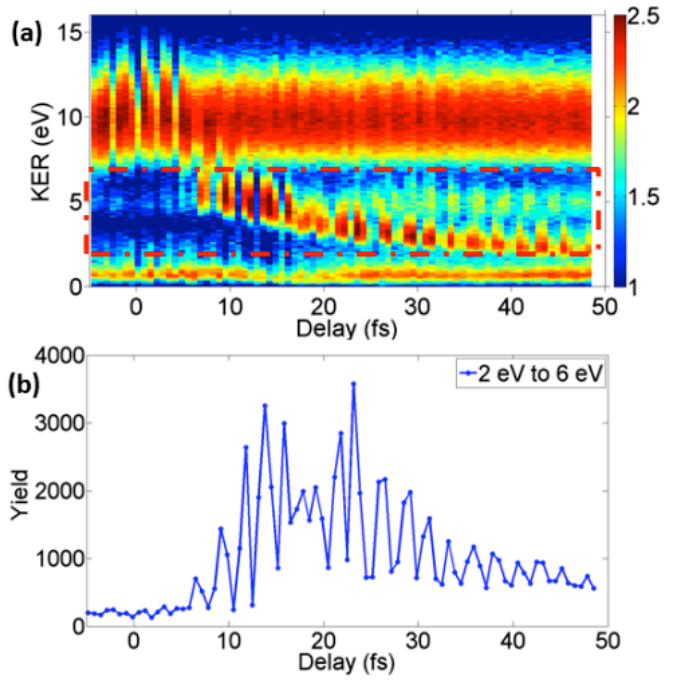

Figure 1. Time-dependent KER spectra (top) and double-proton coincidence integrated yield (bottom) following ionization of $\mathrm{H}_{2}$ by pump pulse.

Acknowledgements This work was funded by Australian Research Council (ARC) under DP110101894. H.X. was supported by an ARC DECRA (DE130101628). D.K. was supported by an ARC Future Fellowship (FT110100513).

\section{REFERENCES}

1. T. Zuo and A.D. Bandrauk, Charge-resonanceenhanced ionization of diatomic molecular ions by intense lasers, Phys. Rev. A 52, R2511-R2514 (1995).

2. E. Constant, H. Stapelfeldt, and P.B. Corkum, Observation of Enhanced Ionization of Molecular Ions in Intense Laser Fields, Phys. Rev. Lett. 76, 4140-4143 (1996)

3. M. Plummer and J.F. McCann, Field-ionization rates of the hydrogen molecular ion, J. Phys. B, 29, 4625-4640 (1996).

4. I. Ben-Itzhak et al., Elusive enhanced ionization structure for $\mathrm{H}_{2}^{+}$in intense ultrashort laser pulses, Phys. Rev. A, 78, 063419 (2009). 